\title{
Status of RI background reduction for SK-Gd
}

\author{
Shintaro Ito* For the Super-Kamiokande Collaboration \\ Okayama University, Faculty of Science, Okayama 700-8530, Japan \\ E-mail: S-itodokayama-u.ac.jp
}

The Super Kamiokande-Gadolinium (SK-Gd) project is the upgrade of the Super-Kamiokande (SK) detector in order to discover Supernova Relic Neutrinos (SRNs) by loading $0.2 \%$ of $\mathrm{Gd}_{2}\left(\mathrm{SO}_{4}\right)_{3}$ into a $50 \mathrm{kton}$ of the SK water tank. In order to continue solar neutrino measurement with low energy threshold at $\sim 3.5 \mathrm{MeV}$, radioactive isotopes (RI) should be reduced before loading. In this talk, the methods to measure $\mathrm{RI}$ in $\mathrm{Gd}_{2}\left(\mathrm{SO}_{4}\right)_{3}$ and the current status of RI background reduction will be presented.

35th International Cosmic Ray Conference - ICRC2017

10-20 July, 2017

Bexco, Busan, Korea

${ }^{*}$ Speaker. 


\section{Introduction}

The Super-Kamiokande (SK) located 1000 m underground at Ikenoyama, Kamioka, Gifu, Japan, is a 50 kton of water Cherenkov detector [四]. The main physics targets for the SK are search for proton decays, and measuring neutrinos from various sources such as the Sun, atmosphere, supernova explosions, artificial neutrino beams, and so on. In order to measure solar neutrinos with low energy threshold at $\sim 3.5 \mathrm{MeV}$, the SK tank is filled with ultra-pure water.

Supernova explosions have been occurring since the beginning of the universe, and neutrinos from these explosions are still floating in our universe. These neutrinos are known as Supernova Relic Neutrinos (SRNs) [వ], however SRNs have not been observed yet. If SRNs are detected, we could study the star formation history of our universe.

On 27th June 2015, new project the Super Kamiokand-Gadolinium (SK-Gd) was approved in the SK Collaboration. The SK-Gd is the upgrade of the SK detector by adding $0.2 \%$ of gadolinium sulfate $\left(\mathrm{Gd}_{2}\left(\mathrm{SO}_{4}\right)_{3}\right)^{1}$ into the SK water tank to discover SRNs world's first [3]]. We aim to introduce $\mathrm{Gd}_{2}\left(\mathrm{SO}_{4}\right)_{3}$ in $2019 \mathrm{JFY}$, and start the SK-Gd experiment.

For solar neutrino analysis, the concentration of radioactive isotopes (RI) in $\mathrm{Gd}_{2}\left(\mathrm{SO}_{4}\right)_{3} \cdot 8 \mathrm{H}_{2} \mathrm{O}$ loaded water must be kept at the same low level as in the current SK water. Thus, $\mathrm{Gd}_{2}\left(\mathrm{SO}_{4}\right)_{3} \cdot 8 \mathrm{H}_{2} \mathrm{O}$ should be purified before loading into the SK water tank not to interfere solar neutrino measurement. Table $\square$ shows our requirements of main RI and typical concentrations in commercial available product. At present, some companies make specially purified $\mathrm{Gd}_{2}\left(\mathrm{SO}_{4}\right)_{3} \cdot 8 \mathrm{H}_{2} \mathrm{O}$ for the SK-Gd. We measured the concentration of radioactive contamination in purified $\mathrm{Gd}_{2}\left(\mathrm{SO}_{4}\right)_{3} \cdot 8 \mathrm{H}_{2} \mathrm{O}$ using two apparatuses, ICP-MS and Ge detector. Features and current status of these two measurements are presented.

Table 1: Our requirements and typical values of commercial available product. All the units are $\mathrm{mBq} / \mathrm{kg}\left(\mathrm{Gd}_{2}\left(\mathrm{SO}_{4}\right)_{3} \cdot 8 \mathrm{H}_{2} \mathrm{O}\right)$.

\begin{tabular}{c|ccc} 
& ${ }^{238} \mathrm{U}$ & ${ }^{232} \mathrm{Th}$ & ${ }^{226} \mathrm{Ra}$ \\
\hline Requirements & 5 & 0.05 & 0.5 \\
Commercial & 50 & 100 & 5
\end{tabular}

\section{ICP-MS measurement}

\subsection{ICP-MS}

ICP-MS stands for Inductively Coupled Plasma-Mass Spectrometry, which can measure the concentration of elements in solutions with high sensitivity of $<10^{-12} \mathrm{~g} / \mathrm{mL}$. Samples introduced into ICP-MS are ionized by argon plasma and then all elements are separated by mass spectrometer, and those ions are quantified. In general, Ge detector is used for short lifetime RI measurement, while ICP-MS is sensitive to long lifetime elements. Therefore, ICP-MS can measure ${ }^{238} \mathrm{U}$ and ${ }^{232} \mathrm{Th}\left(4.5 \times 10^{9}\right.$ and $1.4 \times 10^{10}$ years of half lifetime respectively) with high precision. While ${ }^{226} \mathrm{Ra}$

\footnotetext{
${ }^{1}$ For high solubility, gadolinium sulfate octahydrate $\left(\mathrm{Gd}_{2}\left(\mathrm{SO}_{4}\right)_{3} \cdot 8 \mathrm{H}_{2} \mathrm{O}\right)$ will be used for the SK-Gd.
} 
has shorter lifetime $\left(1.6 \times 10^{4}\right.$ years) than $\mathrm{U}$ and $\mathrm{Th}$, so measurement of $\mathrm{Ra}$ and cross check of ICP-MS measurement for $\mathrm{U}$ and Th were performed by Ge detector.

In December 2016, the ICP-MS "Agilent 7900" was installed into Kamioka clean room in the SK area. Figure $\square$ shows the picture of the ICP-MS [ [4]. This ICP-MS has high sensitivity of $\sim 10^{-14} \mathrm{~g} / \mathrm{mL}$ for $\mathrm{U}$ and $\mathrm{Th}$ in $\mathrm{HNO}_{3}$ solution. The Kamioka clean room was designed as class 1000. For cleaner environment, the auto-sampler is fully covered by the clean booth which is designed as class 100 .

Although using the high sensitive ICP-MS, there are mainly two problems that affect sensitivity of the ICP-MS. These are classified as spectrum and non-spectrum interferences. The former is caused by polyatomic ions that have the similar mass number with target elements, and the later is induced from high concentration matrix element, which is generally called "matrix effects". Figure $\square$ shows the typical example of matrix effects by $\mathrm{Gd}_{2}\left(\mathrm{SO}_{4}\right)_{3} \cdot 8 \mathrm{H}_{2} \mathrm{O}$.

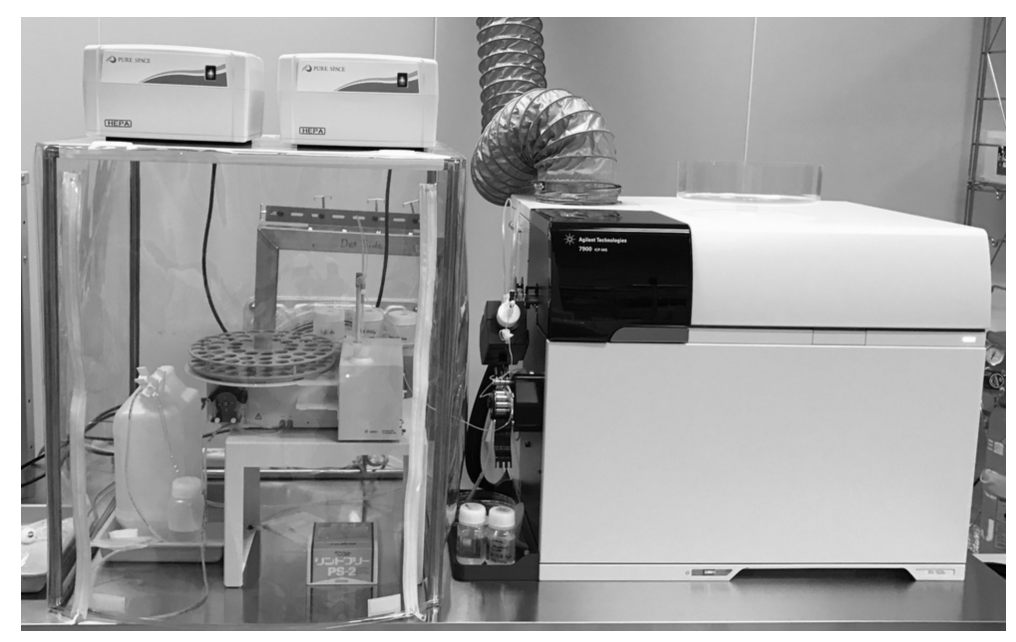

Figure 1: Picture of the ICP-MS “Agilent7900" in Kamioka clean room.

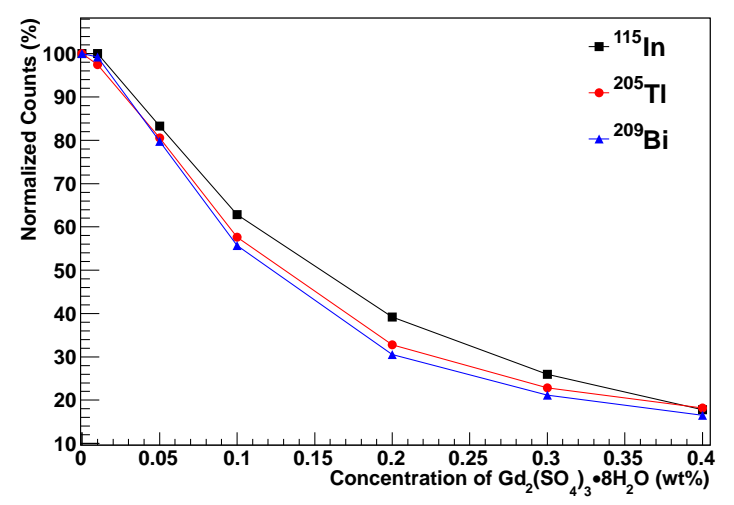

Figure 2: Matrix effects to ${ }^{115} \mathrm{In},{ }^{205} \mathrm{Tl}$, and ${ }^{209} \mathrm{Bi}$ by $\mathrm{Gd}_{2}\left(\mathrm{SO}_{4}\right)_{3} \cdot 8 \mathrm{H}_{2} \mathrm{O}$. Horizontal axis shows concentration of $\mathrm{Gd}_{2}\left(\mathrm{SO}_{4}\right)_{3} \cdot 8 \mathrm{H}_{2} \mathrm{O}$ (wt\%), and vertical axis represents normalized counts. The numbers of counts for ${ }^{115} \mathrm{In}$, ${ }^{205} \mathrm{Tl}$, and ${ }^{209} \mathrm{Bi}$ are normalized to the number of counts for each element in $2 \%$ of $\mathrm{HNO}_{3}$ blank solution. 


\subsection{Development of solid-phase extraction}

In order to reduce interferences from $\mathrm{Gd}_{2}\left(\mathrm{SO}_{4}\right)_{3} \cdot 8 \mathrm{H}_{2} \mathrm{O}$, we developed solid-phase extraction for $\mathrm{U}$ and $\mathrm{Th}$ in $\mathrm{Gd}_{2}\left(\mathrm{SO}_{4}\right)_{3} \cdot 8 \mathrm{H}_{2} \mathrm{O}$. The UTEVA resin is commercial available product of Eichrom Technologies LLC [5], and has been widely used for a variety of analytical separation. All the tetravalent actinides and $\mathrm{U}(\mathrm{VI})$ have strong retention with the UTEVA resin in $>5 \mathrm{M} \mathrm{HNO}_{3}$. On the other hand, Gd is trivalent ion so that Th and $\mathrm{U}$ can be extracted using the UTEVA resin. Thus, the UTEVA resin was used for this study.

Figure $[3$ shows diagram of the developed solid-phase extraction. $0.1 \mathrm{~g}$ of the UTEVA resin was loaded into column with $30 \mathrm{~mL}$ of volume and $8 \mathrm{~mm}$ of inside diameter. For high recovery of Th, the columns were set to two steps as shown in Figure $⿴$. In general, U and Th in the UTEVA resin can be eluted by diluted $\mathrm{HNO}_{3}$ solution. However, Th has high adsorptivity in low concentration acid solution so that the efficiency of elution by only diluted $\mathrm{HNO}_{3}$ is not enough. Therefore, elution procedure was improved as follows: loading $10 \mathrm{~mL}$ of $0.1 \mathrm{M} \mathrm{HNO}_{3}$ to break chemical band between the resin and $\mathrm{U} / \mathrm{Th}$ ( $\mathrm{U}$ is eluted more than $90 \%$ in this procedure), $10 \mathrm{~mL}$ of $7 \mathrm{M}$ $\mathrm{HNO}_{3}$ to collect Th on the columns or surface of the resin, and $10 \mathrm{~mL}$ of $0.1 \mathrm{M} \mathrm{HNO}_{3}$ to collect the remaining Th. These eluted solutions were collected in the same bottle $(30 \mathrm{~mL}$ in total $)$ and measured using the ICP-MS. By this method, Gd was reduced to about $1 / 30$, and about $95 \%$ of $\mathrm{U}$ and $90 \%$ of Th could be extracted from $\mathrm{Gd}_{2}\left(\mathrm{SO}_{4}\right)_{3} \cdot 8 \mathrm{H}_{2} \mathrm{O}$.

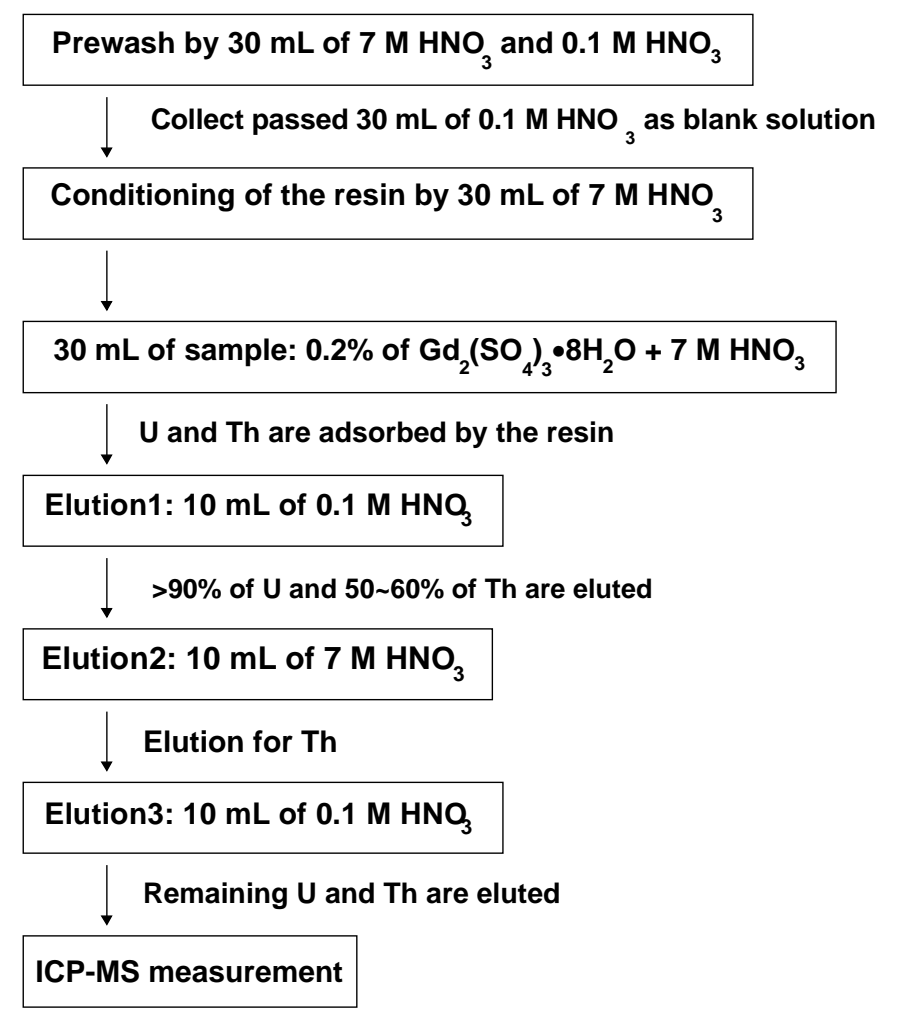

Figure 3: Diagram of the developed solid-phase extraction. 


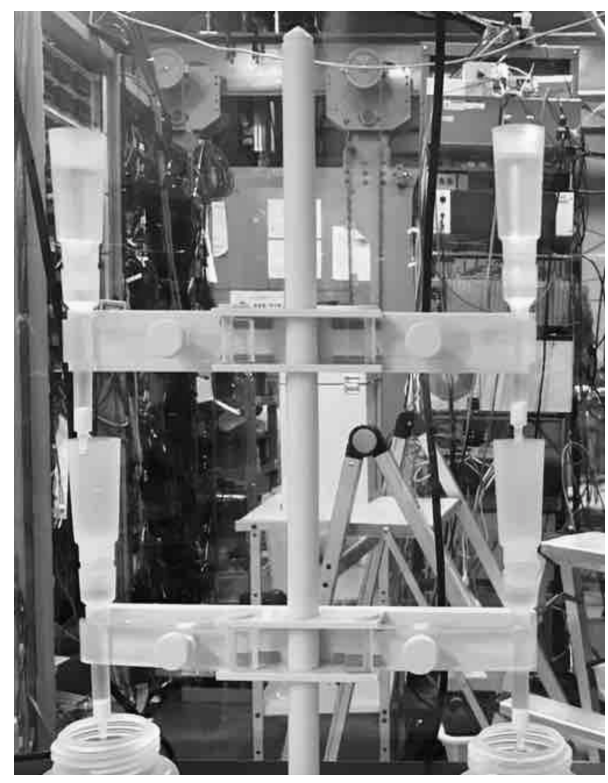

Figure 4: Picture of the experimental setup.

\section{Ge detector measurement}

Ge detector is more sensitive to isotopes from decay chain of ${ }^{238} \mathrm{U}$ and ${ }^{232} \mathrm{Th}$ because of their shorter lifetimes. Measurement of the concentration of Ra and cross check for the ICPMS measurement were performed by high sensitive Ge detector located in Canfranc Underground Laboratory in Spain $[\square]$. Figure $\$$ shows picture of the Canfranc Ge detector farm and Figure 6 shows one of the Ge detector outside its shielding. The Ge detectors in Canfranc are p-type close-end coaxial high purity produced by Canberra France [ [8].

The energy resolution of the Ge detector is about $2 \mathrm{keV}$ (FWHM) at the ${ }^{60} \mathrm{Co}$ gamma line of $1332 \mathrm{keV}$. The active volume of crystals ranges is from 410 to $420 \mathrm{~cm}^{3}$, with about $81 \mathrm{~cm}$ of diameter and height for cylindrical shape. Cryostats are made of ultra-low background aluminum. Each detector is shielded by $5 \mathrm{~cm}$ of oxygen-free copper and $20 \mathrm{~cm}$ of very low activity lead having $<30 \mathrm{mBq} / \mathrm{kg}$ of ${ }^{210} \mathrm{~Pb}$. Nitrogen gas is flushed inside a methacrylate box to avoid airborne radon intrusion.

\section{Results and summary}

Preliminary results of the ICP-MS and the Ge measurements are shown in Table $\square$. By development of the solid-phase extraction, $\mathrm{U}$ and Th in $\mathrm{Gd}_{2}\left(\mathrm{SO}_{4}\right)_{3} \cdot 8 \mathrm{H}_{2} \mathrm{O}$ could be measured by the ICP-MS measurement with high precision. The results of the ICP-MS and the Ge detector are consistent with each other.

By efforts of companies, ${ }^{238} \mathrm{U}$ was well purified by an order of magnitude smaller than our requirement. The concentration of ${ }^{226} \mathrm{Ra}$ was also reduced to almost or less than the value of the goal. While ${ }^{232} \mathrm{Th}$ chain should be removed by a factor of 4 or more than the current values. 


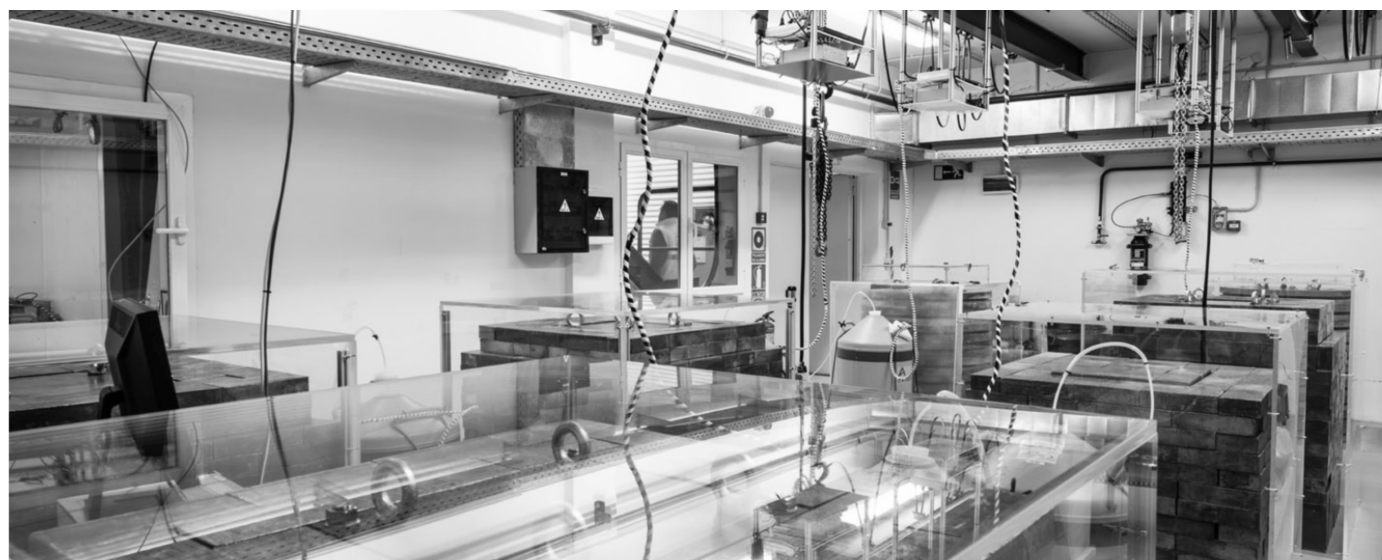

Figure 5: Picture of overall view for the Canfranc Ge detector farm.

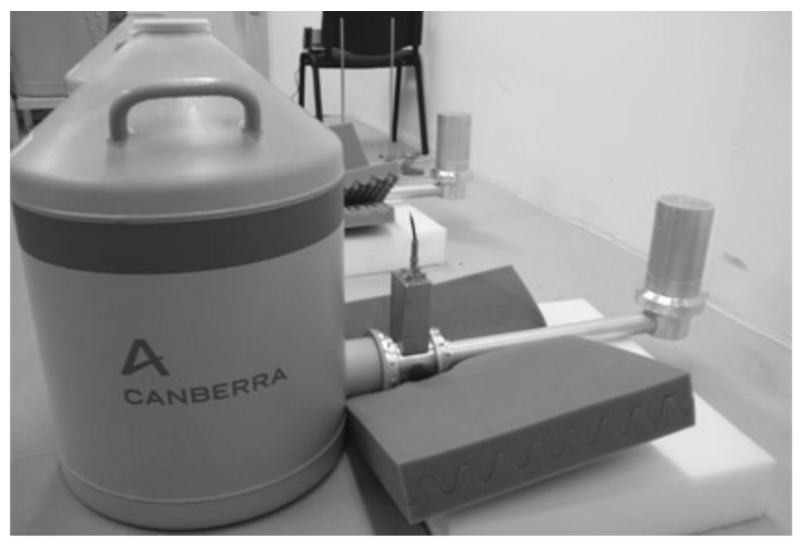

Figure 6: Picture of the Ge detector without shielding in Canfranc.

More purification of $\mathrm{Gd}_{2}\left(\mathrm{SO}_{4}\right)_{3} \cdot 8 \mathrm{H}_{2} \mathrm{O}$ by companies is in progress. For more precise $\mathrm{Th}$ measurement by the ICP-MS, we are now improving the solid-phase extraction to achieve recovery of up to $\sim 100 \%$ and more efficient separation between $\mathrm{Gd}$ and Th.

\section{Acknowledgments}

This work was supported by Grant-in-Aid for Scientific Research on Innovative Areas No. 26104007 and JSPS KAKENHI Grants No. 17K14290.

\section{References}

[1] S. Fukuda et al., Nucl. Instrum. Methods. A501, (2003), 418.

[2] S. Horiuchi et al., Phys. Rev. D79, 083013 (2009).

[3] John F. Beacom and Mark R. Vagins, Phys. Rev. Lett. 93, 171101 (2004).

[4] Agilent Technologies HP.

https://www.agilent.com/en-us/products/icp-ms/icp-ms-systems/7900-icp-ms 
Table 2: Preliminary results of the RI measurements by the ICP-MS and the Ge detector. All the units are $\mathrm{mBq} / \mathrm{kg}\left(\mathrm{Gd}_{2}\left(\mathrm{SO}_{4}\right)_{3} \cdot 8 \mathrm{H}_{2} \mathrm{O}\right)$.

\begin{tabular}{c||c|c|c||c|c|c}
\multicolumn{1}{c||}{ Chain } & \multicolumn{3}{c||}{${ }^{238} \mathrm{U}$} & \multicolumn{3}{c}{${ }^{232} \mathrm{Th}$} \\
\hline \hline Isotope & \multicolumn{2}{c|}{${ }^{238} \mathrm{U}$} & ${ }^{226} \mathrm{Ra}$ & ${ }^{232} \mathrm{Th}$ & ${ }^{228} \mathrm{Ra}$ & ${ }^{228} \mathrm{Th}$ \\
\hline Goal & \multicolumn{2}{|c}{} & $<0.5$ & $<0.05$ & $<0.05$ & $<0.05$ \\
\hline Detector & $\mathrm{Ge}$ & $\mathrm{ICP}-\mathrm{MS}$ & $\mathrm{Ge}$ & $\mathrm{ICP}-\mathrm{MS}$ & $\mathrm{Ge}$ & $\mathrm{Ge}$ \\
\hline Sample A & $<13$ & 0.7 & $0.7 \pm 0.4$ & 1.3 & $<0.4$ & $1.7 \pm 0.4$ \\
\hline Sample B & $<20$ & 0.2 & $<0.6$ & 0.2 & $<0.7$ & $0.5 \pm 0.2$ \\
\hline Sample C & $<9$ & 0.1 & $<0.3$ & 0.2 & $<0.3$ & $<0.4$
\end{tabular}

[5] Eichrom HP. http://www.eichrom.com/products/info/uteva_resin.aspx

[6] E.P. Howritz et al., Analytica Chemca Acta. 266 (1992).

[7] A. Ianni, J.Phys.Conf.Ser. 718 (2016) no.4, 042030

[8] Canberra HP. http://www.canberra.com/ 